\title{
Sums of powers of Fibonacci and Lucas numbers: A new bottom-up approach
}

\author{
Robert Frontczak* \\ Landesbank Baden-Wuerttemberg \\ Am Hauptbahnhof 2, 70173 Stuttgart, Germany \\ e-mail: robert. frontczakalbbw. de
}

Received: 3 October 2017

Accepted: 7 May 2018

\begin{abstract}
We derive expressions for sums of first, second, third and fourth powers of Fibonacci and Lucas numbers and their alternating versions. On our way of exploration we rediscover some known results and present new. Focusing on third and fourth order power sums, our findings complete those of Clary and Hemenway, Melham and Adegoke.
\end{abstract}

Keywords: Fibonacci number, Lucas number, Sums of powers.

2010 Mathematics Subject Classification: 11B37, 11B39.

\section{Introduction}

The Fibonacci numbers $F_{n}$ and Lucas numbers $L_{n}$ satisfy the relations $F_{n}=F_{n-1}+F_{n-2}$ and $L_{n}=L_{n-1}+L_{n-2}$, respectively, with initial conditions $F_{0}=0, F_{1}=1$ and $L_{0}=2, L_{1}=1$. Their Binet forms equal

$$
F_{n}=\frac{\alpha^{n}-\beta^{n}}{\alpha-\beta}, \quad L_{n}=\alpha^{n}+\beta^{n}, \quad n \geq 0,
$$

where $\alpha$ and $\beta$ are roots of the quadratic equation $x^{2}-x-1=0$.

The evaluation of sums of powers of these sequences is a challenging issue. Two pretty examples are

$$
\sum_{k=1}^{n} F_{k}^{2}=F_{n} F_{n+1},
$$

${ }^{*}$ Disclaimer: Statements and conclusions made in this article are entirely those of the author. They do not necessarily reflect the views of LBBW. 
and the cubic formulas derived by Clary and Hemenway [3]

$$
\sum_{k=1}^{n} F_{2 k}^{3}= \begin{cases}\frac{1}{4} F_{n}^{2} L_{n+1}^{2} F_{n-1} L_{n+2} & \text { if } n \text { is even } \\ \frac{1}{4} L_{n}^{2} F_{n+1}^{2} L_{n-1} F_{n+2} & \text { if } n \text { is odd }\end{cases}
$$

and

$$
\sum_{k=1}^{n} F_{4 k}^{3}=\frac{1}{8} F_{2 n}^{2} F_{2 n+2}^{2}\left(L_{4 n+2}+6\right) .
$$

These sums (alternating and/or non-alternating forms) are also studied by Melham [6], Kilic et al. [5] and in two recently published articles by Adegoke [1, 2]. The results obtained, as beautiful as they are, still leave some gaps, which we attempt to fill in this note. Building on a new bottom-up approach, we derive closed-form expressions for sums of first, second, third and fourth powers of Fibonacci and Lucas numbers. We consider both non-alternating and alternating variants.

\section{The key identity}

Our results are based on the following telescoping identities:

Theorem 2.1. Let $f(k)$ be a real sequence and $m, n$ and $j$ be positive integers. Then

$$
\sum_{k=1}^{n}[f(m(k+j))-f(m(k-j))]=\sum_{k=n+1-j}^{n+j} f(m k)-\sum_{k=1-j}^{j} f(m k)
$$

and

$\sum_{k=1}^{n}(-1)^{k-1}[f(m(k+j))-f(m(k-j))]=\sum_{k=n+1-j}^{n+j}(-1)^{k+j-1} f(m k)-\sum_{k=1-j}^{j}(-1)^{k+j-1} f(m k)$.

Especially, for $j=1$

$$
\sum_{k=1}^{n}[f(m(k+1))-f(m(k-1))]=f(m(n+1))+f(m n)-f(m)-f(0),
$$

and

$\sum_{k=1}^{n}(-1)^{k-1}[f(m(k+1))-f(m(k-1))]=(-1)^{n+1} f(m(n+1))+(-1)^{n} f(m n)+f(m)-f(0)$.

Proof. We have

$$
\begin{aligned}
& \sum_{k=1}^{n}[f(m(k+j))-f(m(k-j))]=\sum_{k=1+j}^{n+j} f(m k)-\sum_{k=1-j}^{n-j} f(m k) \\
& =\sum_{k=1-j}^{n+j} f(m k)-\sum_{k=1-j}^{j} f(m k)-\left[\sum_{k=1-j}^{n+j} f(m k)-\sum_{k=n-j+1}^{n+j} f(m k)\right]
\end{aligned}
$$

This proves the first identity. The second one is proved similarly. 


\section{Applications to Fibonacci and Lucas sums}

\subsection{First-order sums}

Proposition 3.1. Let $m$ and $n$ be any positive integers. Then

$$
\sum_{k=1}^{n} F_{2 m k}=\frac{1}{F_{2 m}}\left[F_{m(n+1)}^{2}+F_{m n}^{2}\right]-\frac{F_{m}}{L_{m}}
$$

and

$$
\sum_{k=1}^{n}(-1)^{k} F_{2 m k}=\frac{(-1)^{n}}{F_{2 m}}\left[F_{m(n+1)}^{2}-F_{m n}^{2}\right]-\frac{F_{m}}{L_{m}} .
$$

Proof. We start with the following identity, that can be found in [4]: For all $k$ and $m$

$$
F_{2 k} F_{2 m}=F_{k+m}^{2}-F_{k-m}^{2} .
$$

Replacing $k$ by $k m$ gives

$$
F_{2 m k} F_{2 m}=F_{m(k+1)}^{2}-F_{m(k-1)}^{2} .
$$

Set $f(k)=F_{k}^{2}$ in Equation (2.3) to get

$$
\sum_{k=1}^{n} F_{2 m k}=\frac{1}{F_{2 m}}\left[F_{m(n+1)}^{2}+F_{m n}^{2}-F_{m}^{2}\right] .
$$

The result follows from the the relation $L_{m}=F_{2 m} / F_{m}$.

The alternating sum is obtained in the same manner from Equation (2.4).

An alternative evaluation of the sum in (3.1) is obtained in the recently published preprint [2] by Adegoke (Lemma 2.2):

$$
\sum_{k=1}^{n} F_{2 m k}= \begin{cases}F_{m n} F_{m(n+1)} / F_{m} & \text { if } m \text { is even } \\ F_{m n} L_{m(n+1)} / L_{m} & \text { if } m \text { is odd and } n \text { is even } \\ L_{m n} F_{m(n+1)} / L_{m} & \text { if } m \text { is odd and } n \text { is odd. }\end{cases}
$$

Concerning the evaluation of the alternating version the author could not find a reference in the literature. A result of similar nature is stated in [5] (Corollary 1), where among others a formula for $\sum_{k=1}^{n}(-1)^{k} F_{(2 m+1) k}$ is derived.

The corresponding identities for Lucas numbers are given in the next Proposition:

Proposition 3.2. Let $m$ and $n$ be any positive integers. Then

$$
\sum_{k=1}^{n} L_{2 m k}=\frac{1}{F_{2 m}}\left[F_{2 m(n+1)}+F_{2 m n}\right]-1
$$

and

$$
\sum_{k=1}^{n}(-1)^{k} L_{2 m k}=\frac{(-1)^{n}}{F_{2 m}}\left[F_{2 m(n+1)}-F_{2 m n}\right]-1 .
$$


Proof. Set $f(k)=F_{2 k+2 m}$ in Equation (2.3) and use

$$
F_{v} L_{u}=F_{u+v}-(-1)^{v} F_{u-v}
$$

with $v=2 m$ and $u=2 m(k+1)$ to get

$$
F_{2 m} L_{2 m(k+1)}=F_{2 m(k+2)}-F_{2 m k} .
$$

This gives

$$
F_{2 m} \sum_{k=1}^{n} L_{2 m(k+1)}=F_{2 m(n+2)}+F_{2 m(n+1)}-F_{4 m}-F_{2 m} .
$$

Since

$$
\sum_{k=1}^{n} L_{2 m(k+1)}=\sum_{k=1}^{n+1} L_{2 m k}-L_{2 m}
$$

the first part follows after replacing $n+1$ by $n$ and using the relation $L_{m}=F_{2 m} / F_{m}$.

The alternating sum is obtained in the same manner from Equation (2.4).

The identities presented in Proposition 3.2 offer a way for a concise treatment of the firstorder Lucas sums. However, they can be modified to obtain more familiar versions known from previous studies: If $m$ is even, then we can use

$$
L_{v} F_{u}=F_{u+v}+(-1)^{v} F_{u-v}
$$

with $v=m$ and $u=2 m n+m$ to get

$$
L_{m} F_{m(2 n+1)}=F_{2 m(n+1)}+F_{2 m n} .
$$

This results in

$$
\sum_{k=1}^{n} L_{2 m k}=\frac{F_{m(2 n+1)}}{F_{m}}-1=\frac{F_{m n} L_{m(n+1)}}{F_{m}} .
$$

These formulas appear in Adegoke [1, Equation (2.4)] and Melham [6, Equation (5.3)]. If $m$ is odd, we can use the similar identity

$$
F_{v} L_{u}=F_{u+v}-(-1)^{v} F_{u-v}
$$

with $v=m$ and $u=2 m n+m$ to get

$$
F_{m} L_{m(2 n+1)}=F_{2 m(n+1)}+F_{2 m n} .
$$

This gives

$$
\sum_{k=1}^{n} L_{2 m k}=\frac{L_{m(2 n+1)}}{L_{m}}-1,
$$

which appears in Adegoke [1, Equation (2.9)]. Melham [6, Equation (2.12)] states the relation as

$$
\sum_{k=1}^{n} L_{2 m k}= \begin{cases}5 F_{m n} F_{m(n+1)} / L_{m} & \text { if } m \text { is odd and } n \text { is even } \\ L_{m n} L_{m(n+1)} / L_{m} & \text { if } m \text { is odd and } n \text { is odd. }\end{cases}
$$


The alternating sum identity (3.8) appears in the article of Kilic et al. [5]. Adegoke [1] and Melham [6] state the identity in different versions: If $m$ is odd, then

$$
\sum_{k=1}^{n}(-1)^{k} L_{2 m k}=(-1)^{n} \frac{F_{m n} L_{m(n+1)}}{F_{m}},
$$

(Adegoke [1, Equation (2.5)] and Melham [6, Equation (5.1)]) and if $m$ is even, then

$$
\sum_{k=1}^{n}(-1)^{k} L_{2 m k}=(-1)^{n} \frac{L_{m(2 n+1)}}{L_{m}}-1 .
$$

\subsection{Second-order sums}

Proposition 3.3. Let $m$ and $n$ be any positive integers.

If $m$ is even, then

$$
\sum_{k=1}^{n} F_{m k}^{2}=\frac{1}{5 F_{2 m}}\left[F_{2 m(n+1)}+F_{2 m n}\right]-\frac{1+2 n}{5} .
$$

If $m$ is odd, then

$$
\sum_{k=1}^{n} F_{m k}^{2}=\frac{1}{5 F_{2 m}}\left[F_{2 m(n+1)}+F_{2 m n}\right]+\frac{(-1)^{n+1}}{5} .
$$

Also, if $m$ is odd, then

$$
\sum_{k=1}^{n}(-1)^{k} F_{m k}^{2}=\frac{(-1)^{n}}{5 F_{2 m}}\left[F_{2 m(n+1)}-F_{2 m n}\right]-\frac{1+2 n}{5},
$$

and if $m$ is even, then

$$
\sum_{k=1}^{n}(-1)^{k} F_{m k}^{2}=\frac{(-1)^{n}}{5 F_{2 m}}\left[F_{2 m(n+1)}-F_{2 m n}\right]+\frac{(-1)^{n+1}}{5} .
$$

Proof. Replacing $m$ by $m k$ in the relation

$$
5 F_{m}^{2}=L_{2 m}+(-1)^{m+1} 2
$$

and summing from $k=1$ to $n$ gives

$$
5 \sum_{k=1}^{n} F_{m k}^{2}=\sum_{k=1}^{n} L_{2 m k}-2 \sum_{k=1}^{n}(-1)^{m k}
$$

and

$$
5 \sum_{k=1}^{n}(-1)^{k} F_{m k}^{2}=\sum_{k=1}^{n}(-1)^{k} L_{2 m k}-2 \sum_{k=1}^{n}(-1)^{(m+1) k} .
$$

Now, both expressions follow essentially from Proposition 3.2 and the observation that

$$
\sum_{k=1}^{n}(-1)^{m k}= \begin{cases}n & \text { if } m \text { is even } \\ \left(-1+(-1)^{n}\right) / 2 & \text { if } m \text { is odd. }\end{cases}
$$


Proposition 3.4. Let $m$ and $n$ be any positive integers.

If $m$ is even, then

$$
\sum_{k=1}^{n} L_{m k}^{2}=\frac{1}{F_{2 m}}\left[F_{2 m(n+1)}+F_{2 m n}\right]-1+2 n .
$$

If $m$ is odd, then

$$
\sum_{k=1}^{n} L_{m k}^{2}=\frac{1}{F_{2 m}}\left[F_{2 m(n+1)}+F_{2 m n}\right]-2+(-1)^{n} .
$$

Also, if $m$ is odd, then

$$
\sum_{k=1}^{n}(-1)^{k} L_{m k}^{2}=\frac{(-1)^{n}}{F_{2 m}}\left[F_{2 m(n+1)}-F_{2 m n}\right]-1+2 n
$$

and finally, if $m$ is even, then

$$
\sum_{k=1}^{n}(-1)^{k} L_{m k}^{2}=\frac{(-1)^{n}}{F_{2 m}}\left[F_{2 m(n+1)}-F_{2 m n}\right]-2+(-1)^{n} .
$$

Proof. Replacing $m$ by $m k$ in the relation

$$
L_{m}^{2}=5 F_{m}^{2}+(-1)^{m} 4,
$$

and summing from $k=1$ to $n$ gives

$$
\sum_{k=1}^{n} L_{m k}^{2}=5 \sum_{k=1}^{n} F_{m k}^{2}+4 \sum_{k=1}^{n}(-1)^{m k}
$$

and

$$
\sum_{k=1}^{n}(-1)^{k} L_{m k}^{2}=5 \sum_{k=1}^{n}(-1)^{k} F_{m k}^{2}+4 \sum_{k=1}^{n}(-1)^{(m+1) k},
$$

The statements follow from Proposition 3.3.

The first four special cases of the quadratic sums are

$$
\begin{gathered}
\sum_{k=1}^{n} F_{k}^{2}=\frac{1}{5}\left[F_{2 n+2}+F_{2 n}+(-1)^{n+1}\right], \\
\sum_{k=1}^{n}(-1)^{k} F_{k}^{2}=\frac{(-1)^{n}}{5}\left[F_{2 n+2}-F_{2 n}\right]-\frac{1+2 n}{5}, \\
\sum_{k=1}^{n} L_{k}^{2}=F_{2 n+2}+F_{2 n}-2+(-1)^{n}
\end{gathered}
$$

and

$$
\sum_{k=1}^{n}(-1)^{k} L_{k}^{2}=(-1)^{n}\left[F_{2 n+2}-F_{2 n}\right]-1+2 n .
$$




\subsection{Third-order sums}

Proposition 3.5. Let $m$ and $n$ be any positive integers. Then

$$
\sum_{k=1}^{n} F_{2 m k}^{3}=\frac{1}{5}\left[\frac{1}{F_{6 m}}\left[F_{3 m(n+1)}^{2}+F_{3 m n}^{2}\right]-\frac{F_{3 m}}{L_{3 m}}\right]-\frac{3}{5}\left[\frac{1}{F_{2 m}}\left[F_{m(n+1)}^{2}+F_{m n}^{2}\right]-\frac{F_{m}}{L_{m}}\right] .
$$

Similarly,

$$
\sum_{k=1}^{n}(-1)^{k} F_{2 m k}^{3}=\frac{(-1)^{n}}{5 F_{6 m}}\left[F_{3 m(n+1)}^{2}-F_{3 m n}^{2}\right]-\frac{3}{5} \frac{(-1)^{n}}{F_{2 m}}\left[F_{m(n+1)}^{2}-F_{m n}^{2}\right]-\frac{F_{3 m}}{5 L_{3 m}}+\frac{3 F_{m}}{5 L_{m}}
$$

Proof. Replacing $m$ by $2 m k$ in the relation

$$
5 F_{m}^{3}=F_{3 m}-3(-1)^{m} F_{m}
$$

and summing from $k=1$ to $n$ gives

$$
5 \sum_{k=1}^{n} F_{2 m k}^{3}=\sum_{k=1}^{n} F_{6 m k}-3 \sum_{k=1}^{n} F_{2 m k}
$$

and

$$
5 \sum_{k=1}^{n}(-1)^{k} F_{2 m k}^{3}=\sum_{k=1}^{n}(-1)^{k} F_{6 m k}-3 \sum_{k=1}^{n}(-1)^{k} F_{2 m k} .
$$

Now, both results follow immediately from Proposition 3.1.

Proposition 3.6. Let $m$ and $n$ be any positive integers. Then

$$
\sum_{k=1}^{n} L_{2 m k}^{3}=\frac{1}{F_{6 m}}\left[F_{6 m(n+1)}+F_{6 m n}\right]+\frac{3}{F_{2 m}}\left[F_{2 m(n+1)}+F_{2 m n}\right]-4 .
$$

Similarly,

$$
\sum_{k=1}^{n}(-1)^{k} L_{2 m k}^{3}=\frac{(-1)^{n}}{F_{6 m}}\left[F_{6 m(n+1)}-F_{6 m n}\right]+\frac{3(-1)^{n}}{F_{2 m}}\left[F_{2 m(n+1)}-F_{2 m n}\right]-4 .
$$

Proof. The proof follows from the identity

$$
L_{m}^{3}=L_{3 m}+3(-1)^{m} L_{m}
$$

and Proposition 3.2.

Our results for the non-alternating cubic sums for $F_{n}$ and/or $L_{n}$ must be seen as variants of the remarkable product evaluations of Adegoke [2] and Clary and Hemenway [3]. In contrast, the evaluations of the alternating counterparts seem to be new. The author could not find a reference for these sums.

For $m=1$ we get the following identities as explicit examples of the results from this section:

$$
\sum_{k=1}^{n} F_{2 k}^{3}=\frac{1}{40}\left[F_{3 n+3}^{2}+F_{3 n}^{2}\right]-\frac{3}{5}\left[F_{n+1}^{2}+F_{n}^{2}\right]+\frac{1}{2}
$$




$$
\begin{gathered}
\sum_{k=1}^{n}(-1)^{k} F_{2 k}^{3}=\frac{(-1)^{n}}{40}\left[F_{3 n+3}^{2}-F_{3 n}^{2}\right]-\frac{3(-1)^{n}}{5}\left[F_{n+1}^{2}-F_{n}^{2}\right]+\frac{1}{2}, \\
\sum_{k=1}^{n} L_{2 k}^{3}=\frac{1}{8}\left[F_{6 n+6}+F_{6 n}\right]+3\left[F_{2 n+2}+F_{2 n}\right]-4,
\end{gathered}
$$

and

$$
\sum_{k=1}^{n}(-1)^{k} L_{2 k}^{3}=\frac{(-1)^{n}}{8}\left[F_{6 n+6}-F_{6 n}\right]+3(-1)^{n}\left[F_{2 n+2}-F_{2 n}\right]-4
$$

\subsection{Fourth-order sums}

The results of this section represent alternative expressions to the product identities of Melham [6] and Adegoke [1]. Instead of choosing a compact form, we write them as separate sums.

Proposition 3.7. Let $m$ and $n$ be any positive integers. Then

$$
25 \sum_{k=1}^{n} F_{m k}^{4}= \begin{cases}F_{4 m}\left[F_{4 m(n+1)}+F_{4 m n}\right]-\frac{4}{F_{2 m}}\left[F_{2 m(n+1)}+F_{2 m n}\right]+3+6 n & \text { if } m \text { is even } \\ \frac{1}{F_{4 m}}\left[F_{4 m(n+1)}+F_{4 m n}\right]-\frac{4(-1)^{n}}{F_{2 m}}\left[F_{2 m(n+1)}-F_{2 m n}\right]+3+6 n & \text { if } m \text { is odd. }\end{cases}
$$

Also, if $m$ is even, then

$$
25 \sum_{k=1}^{n}(-1)^{k} F_{m k}^{4}= \begin{cases}\frac{1}{F_{4 m}}\left[F_{4 m(n+1)}-F_{4 m n}\right]-\frac{4}{F_{2 m}}\left[F_{2 m(n+1)}-F_{2 m n}\right]+3 & \text { if } n \text { is even } \\ \frac{-1}{F_{4 m}}\left[F_{4 m(n+1)}-F_{4 m n}\right]+\frac{4}{F_{2 m}}\left[F_{2 m(n+1)}-F_{2 m n}\right]-3 & \text { if } n \text { is odd. }\end{cases}
$$

Finally, if $m$ is odd, then

$$
25 \sum_{k=1}^{n}(-1)^{k} F_{m k}^{4}= \begin{cases}\frac{1}{F_{4 m}}\left[F_{4 m(n+1)}-F_{4 m n}\right]-\frac{4}{F_{2 m}}\left[F_{2 m(n+1)}+F_{2 m n}\right]+3 & \text { if } n \text { is even } \\ \frac{-1}{F_{4 m}}\left[F_{4 m(n+1)}-F_{4 m n}\right]-\frac{4}{F_{2 m}}\left[F_{2 m(n+1)}+F_{2 m n}\right]-3 & \text { if } n \text { is odd. }\end{cases}
$$

Proof. Squaring the identity

$$
5 F_{m}^{2}=L_{2 m}-(-1)^{m} 2,
$$

replacing $m$ by $m k$ and summing from $k=1$ to $n$ gives

$$
25 \sum_{k=1}^{n} F_{m k}^{4}= \begin{cases}\sum_{k=1}^{n} L_{2 m k}^{2}-4 \sum_{k=1}^{n} L_{2 m k}+4 n & \text { if } m \text { is even } \\ \sum_{k=1}^{n} L_{2 m k}^{2}-4 \sum_{k=1}^{n}(-1)^{k} L_{2 m k}+4 n & \text { if } m \text { is odd. }\end{cases}
$$

The first formula follows from Propositions 3.4 and 3.2.

To establish the alternating versions, we first observe that

$$
25 \sum_{k=1}^{n}(-1)^{k} F_{m k}^{4}= \begin{cases}\sum_{k=1}^{n}(-1)^{k} L_{2 m k}^{2}-4 \sum_{k=1}^{n} L_{2 m k}+4 \sum_{k=1}^{n}(-1)^{k} & \text { if } m \text { is odd } \\ \sum_{k=1}^{n}(-1)^{k} L_{2 m k}^{2}-4 \sum_{k=1}^{n}(-1)^{k} L_{2 m k}+4 \sum_{k=1}^{n}(-1)^{k} & \text { if } m \text { is even. }\end{cases}
$$

The last sums equal 0 or -1 depending on the parity of $n$. This leads to four different $(m, n)$ combinations. The final results follow again from Propositions 3.4 and 3.2. 
Proposition 3.8. Let $m$ and $n$ be any positive integers. Then

$$
\sum_{k=1}^{n} L_{m k}^{4}= \begin{cases}\frac{1}{F_{4 m}}\left[F_{4 m(n+1)}+F_{4 m n}\right]+\frac{4}{F_{2 m}}\left[F_{2 m(n+1)}+F_{2 m n}\right]-5+6 n & \text { if } m \text { is even } \\ \frac{1}{F_{4 m}}\left[F_{4 m(n+1)}+F_{4 m n}\right]+\frac{4(-1)^{n}}{F_{2 m}}\left[F_{2 m(n+1)}-F_{2 m n}\right]-5+6 n & \text { if } m \text { is odd. }\end{cases}
$$

Also, if $m$ is even, then

$$
\sum_{k=1}^{n}(-1)^{k} L_{m k}^{4}= \begin{cases}\frac{1}{F_{4 m}}\left[F_{4 m(n+1)}-F_{4 m n}\right]+\frac{4}{F_{2 m}}\left[F_{2 m(n+1)}-F_{2 m n}\right]-5 & \text { if } n \text { is even } \\ \frac{-1}{F_{4 m}}\left[F_{4 m(n+1)}-F_{4 m n}\right]-\frac{4}{F_{2 m}}\left[F_{2 m(n+1)}-F_{2 m n}\right]-11 & \text { if } n \text { is odd. }\end{cases}
$$

Finally, if $m$ is odd, then

$$
\sum_{k=1}^{n}(-1)^{k} L_{m k}^{4}= \begin{cases}\frac{1}{F_{4 m}}\left[F_{4 m(n+1)}-F_{4 m n}\right]+\frac{4}{F_{2 m}}\left[F_{2 m(n+1)}+F_{2 m n}\right]-5 & \text { if } n \text { is even } \\ \frac{-1}{F_{4 m}}\left[F_{4 m(n+1)}-F_{4 m n}\right]+\frac{4}{F_{2 m}}\left[F_{2 m(n+1)}+F_{2 m n}\right]-11 & \text { if } n \text { is odd. }\end{cases}
$$

Proof. Squaring the identity

$$
L_{m}^{2}=L_{2 m}+(-1)^{m} 2,
$$

replacing $m$ by $m k$ and summing from $k=1$ to $n$ gives

$$
\sum_{k=1}^{n} L_{m k}^{4}= \begin{cases}\sum_{k=1}^{n} L_{2 m k}^{2}+4 \sum_{k=1}^{n} L_{2 m k}+4 n & \text { if } m \text { is even } \\ \sum_{k=1}^{n} L_{2 m k}^{2}+4 \sum_{k=1}^{n}(-1)^{k} L_{2 m k}+4 n & \text { if } m \text { is odd. }\end{cases}
$$

and

$$
\sum_{k=1}^{n}(-1)^{k} L_{m k}^{4}= \begin{cases}\sum_{k=1}^{n}(-1)^{k} L_{2 m k}^{2}+4 \sum_{k=1}^{n} L_{2 m k}+4 \sum_{k=1}^{n}(-1)^{k} & \text { if } m \text { is odd } \\ \sum_{k=1}^{n}(-1)^{k} L_{2 m k}^{2}+4 \sum_{k=1}^{n}(-1)^{k} L_{2 m k}+4 \sum_{k=1}^{n}(-1)^{k} & \text { if } m \text { is even. }\end{cases}
$$

The identities follow again from Propositions 3.4 and 3.2.

We conclude with four explicit examples:

$$
\begin{gathered}
25 \sum_{k=1}^{n} F_{k}^{4}=\frac{1}{3}\left[F_{4 n+4}+F_{4 n}\right]-4(-1)^{n}\left[F_{2 n+2}-F_{2 n}\right]+3+6 n, \\
25 \sum_{k=1}^{n}(-1)^{k} F_{k}^{4}= \begin{cases}\frac{1}{3}\left[F_{4 n+4}-F_{4 n}\right]-4\left[F_{2 n+2}+F_{2 n}\right]+3 & \text { if } n \text { is even } \\
-\frac{1}{3}\left[F_{4 n+4}-F_{4 n}\right]-4\left[F_{2 n+2}+F_{2 n}\right]-3 & \text { if } n \text { is odd, }\end{cases} \\
\sum_{k=1}^{n} L_{k}^{4}=\frac{1}{3}\left[F_{4 n+4}+F_{4 n}\right]+4(-1)^{n}\left[F_{2 n+2}-F_{2 n}\right]-5+6 n,
\end{gathered}
$$

and

$$
\sum_{k=1}^{n}(-1)^{k} L_{k}^{4}= \begin{cases}\frac{1}{3}\left[F_{4 n+4}-F_{4 n}\right]+4\left[F_{2 n+2}+F_{2 n}\right]-5 & \text { if } n \text { is even } \\ -\frac{1}{3}\left[F_{4 n+4}-F_{4 n}\right]+4\left[F_{2 n+2}+F_{2 n}\right]-11 & \text { if } n \text { is odd. }\end{cases}
$$




\section{References}

[1] Adegoke, K., Sums of fourth powers of Fibonacci and Lucas numbers, Preprint, May 2017, Available via arXiv.

[2] Adegoke, K., Factored closed-form expressions for the sums of cubes of Fibonacci and Lucas numbers, Preprint, June 2017, Available via arXiv.

[3] Clary, S. \& Hemenway, P. D. (1993) On sums of cubes of Fibonacci numbers, in Applications of Fibonacci Numbers, Kluwer Academic Publishers, 123-136.

[4] Daykin, D. E. \& Dresel, L. A. G. (1967) Identities for products of Fibonacci and Lucas numbers, The Fibonacci Quarterly, 5 (4), 367-370.

[5] Kilic, E., Omur, N. \& Ulutas, Y. (2011) Alternating sums of the powers of Fibonacci and Lucas numbers, Miskolc Mathematical Notes, 12 (1), 87-103.

[6] Melham, R.S. (2000) Alternating sums of fourth powers of Fibonacci and Lucas numbers, The Fibonacci Quarterly, 38 (3), 254-259. 